\title{
Folded Additive Structures: A Nonpolynomial Model of Some Factorial Interactions 1
}

\author{
Paul E. LehNer \\ University of Michigan
}

\begin{abstract}
A nonpolynomial measurement model for representing nonadditive compositions of stimulus dimensions is presented. This representation, which is referred to as a folded additive structure, permits the decomposition of a behavioral ordering into independent factors in some of the cases where the behavior itself does not directly reflect this independence. Psychologically, this representation corresponds to a model of behavior which assumes that (1) the effect stimulus attributes have on behavior is mediated by a single relevent psychological dimension, (2) the behavioral ordering is single-peaked over this mediating dimension and (3) the mediating dimension has a conjoint additive ordering over a set of relevent stimulus dimensions. Necessary and sufficient tests for folded additive structures are given. In addition, five psychological theories which make these assumptions are discussed.
\end{abstract}

\section{INTRODUCTION}

It is characteristic of all sciences that whenever multiple explanations of an event exist, the simplest or most parsimonious one is preferred. In the behavioral sciences this tendency is exhibited in part by the ubiquitous use of behavioral models which assume that various attributes of a stimulus environment have an additive effect on behavior. Consider, for instance, the frequent use of additive conjoint measurement and multiple regression techniques, both of which attempt to fit simple additive models to data.

Despite the fact that such simple additive models are widely used, they are by nature very limited. More often than not, it has been the behavioral scientist's experience that environmental factors interact in a nonadditive manner.

In this paper, a theory of nonadditive interactions is examined. This theory, which is called folded additivity, ${ }^{2}$ provides a mathematical model that decomposes a behavioral ordering into independent factors in some of the cases where the behavior itself does not directly reflect this independence. That is, we provide a simple model that can account for some complex empirical structures which violate additivity. A second more general model, called folded independence, is also presented.

\footnotetext{
' This research was supported by NSF Grant BNS78-09101. Please send reprint requests to: Paul E. Lehner, Department of Psychology, University of Michigan, Ann Arbor, Mich. 48109.

${ }^{2}$ The concept of folded additivity was first presented to me in a class I took from Dr. Clyde $\mathrm{H}$. Coombs in 1977 at the University of Michigan.
} 
Assume that we have an ordering, $\geqslant_{0}$, on a set of elements, $D$, which varies on several dimensions, $A_{1}, A_{2}, \ldots, A_{n}$, i.e., $D=A_{1} X A_{2} X \cdots X A_{n}$.

The system $\left\langle D, \geqslant_{0}\right\rangle$ is said to have to have a folded additive representation if there exist real-valued functions $G$ and $F$ on $D$, and a function $r_{i}$ on each $A_{i}$, such that the following three properties are satisfied:

(P1) The numerical ordering that $G$ induces on the elements of $D$ is the same as that of $\geqslant_{0}$.

(P2) The ordering that $G$ induces on $D$ is single-peaked, (i.e., contains only one local maximum) over the ordering that $F$ induces on $D$.

(P3) $F$ is an aditive composition of the dimensions of $D$, that is,

$$
F\left(a_{1}, a_{2}, \ldots, a_{n}\right)=r_{1}\left(a_{1}\right)+r_{2}\left(a_{2}\right)+\cdots+r_{n}\left(a_{n}\right),
$$

for all $a_{i}$ in $A_{i}$.

The system $\left\langle D, \geqslant_{0}\right\rangle$ is said to have a folded independent representation if $\mathrm{P} 1$ and $\mathrm{P} 2$ hold and $\mathrm{P} 3$ is replaced by the following more general property.

$\left(\mathrm{P}^{\prime}\right) \quad F$ is a monotonic decomposition of the dimensions of $D$, that is,

$$
F\left(a_{1}, a_{2}, \ldots, a_{n}\right)=f\left(r_{1}\left(a_{1}\right), r_{2}\left(a_{2}\right), \ldots, r_{n}\left(a_{n}\right),\right.
$$

where $f$ increase monotonically with each of its arguments.

One way to represent a folded additive structure with two factors is by a set of indifference curves on the two-dimensional space defined by the stimulus factors. A folded additive structure implies that the stimulus dimensions can be scaled in such a way that there exist a set of negatively sloped parallel lines for which (1) the points on each line form an equivalence class and (2) there is a single-peaked function over these equivalence classes. The line corresponding to the equivalence class of points highest in the behavioral ordering is called the ideal trace (Coombs \& Meyer, 1969).

In Fig. 1, for instance, we can see that $A$ is closer to the ideal trace than is $B$ or $C$, which are on the same line, while $D$ is even farther away. By the single-peakedness property, a behavioral ordering, $\geqslant_{0}$, that corresponds to this figure would have

$$
A>{ }_{0} B={ }_{0} C>{ }_{0} D .
$$

If, on the other hand, the $B-C$ line was the ideal trace, rather than the line indicated, then single-peakedness would imply

$$
B \text { and } C>_{0} A \text { and } D .
$$

Note that in this last inequality, the orderings on the two dimensions are not mutually independent. Consequently, this behavioral ordering could not have an additive representation.

Generally speaking, both folded additivity and folded independence correspond to a model of behavior which makes the following three psychological assumptions. 

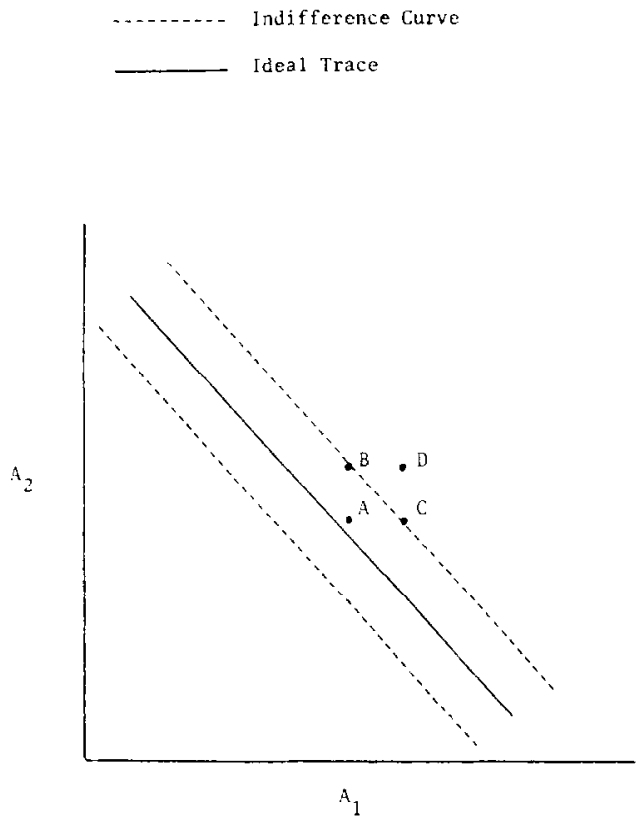

F1G. 1. Indifference curves in a two-dimensional folded additive space.

(A1) The effect of the stimulus attributes on behavior is mediated by a single relevent emergent dimension.

(A2) The behavioral ordering is single-peaked over this mediating dimension.

(A3) The ordering on the mediating dimension can be decomposed into independent factors.

In the case of folded additivity this decomposition is an additive one.

These assumptions can be found in a wide variety of behavioral contexts. A first example can be seen in some decision theory research. Specifically, Coombs' $(1969,1975)$ Portfolio theory predicts that, whenever expected value is held constant, the preference order on a set of gambles is mediated solely by the perceived riskiness of each of the gambles, where the preference order is single-peaked over the risk order. Furthermore, under certain conditions, perceived riskiness is thought to be an additive function of variance and skewness (Coombs \& Lehner, 1980; note 1), and variance and number of plays (Coombs \& Huang, 1970; Coombs \& Meyer, 1969). Consequently, Portfolio theory would predict that the preference order on a set of gambles which varied on either of these pairs of dimensions would satisfy folded additivity. In fact, in Coombs and Meyer (1969) and Lehner (1980) many of the necessary conditions for folded additivity were explicitly tested.

Further examples are to be found in the class of theories which suggest that task performance is single-peaked over arousal, the inverted-U hypothesis 
(Bargh \& Cohen, 1978; Innes \& Young, 1975; Katahn, Blanton, \& Gipson, 1967; Martens \& Landers, 1972; Sorce \& Fouts, 1973; Stennet, 1957). According to this hypothesis, there exists a general dimension of task-oriented arousal for which a certain level of arousal facilitates optimal performance and for which greater or lesser levels result in poorer performance. When this hypothesis is tested (usually in the context of social facilitation research) it is frequently the case that several, hypothetically independent, sources of arousal are manipulated in a factorial design (see, for example, Innes \& Young, 1975; Leibling \& Shaver, 1973). If these sources of arousal are combined into a single arousal dimension, which mediates performance without respect to its component sources, then we are dealing with folded independence. On the other hand, if the independent variables each have a separable effect on performance, then we would expect some of the necessary conditions for folded independence to be violated.

Another context in which folded independence may be seen is in some research on interpersonal attraction. In particular, Aronson, Willerman, and Floyd (1966) have found that the attractiveness of a highly competent or superior person is increased when he makes a blunder, while a similar blunder decreases the attractiveness of a person of less ability. Aronson et al. explained this behavior as follows,

\footnotetext{
A near perfect or superior individual who shows that he is capable of an occasional blunder or pratfall may come to be regarded as more human and more approachable; consequently he will be liked better because of his pratfall. On the other hand, if a mediocre or average person commits an identical blunder, he will not undergo an increase in attractiveness. Indeed, since it would suggest that he is very mediocre, it should lower his attractiveness (p. 227).
}

In other words, liking for other people is diminished by deviation from a norm or reference point on a general ability dimension. A pratfall has the effect of reducing a person's level of perceived ability. Consequently, committing a pratfall may bring a superior person nearer to that reference point, but move a mediocre person away from it. If this interpretation is correct, then we would expect other sources of perceived ability to have a similar effect. For example, graduating from college may make a person more likable to some and less likable to others.

A fourth context is political voting behavior. Political scientists often make the simplifying assumption that a voter's preference for a political candidate is determined by the candidates location, relative to that of the voter, on a general liberal-conservative dimension. That is, each voter has a single-peaked preference function over the liberal-conservative dimension, (e.g., Arrow, 1963, Chap. 7). In addition, it is reasonable to assume that a candidate's overall level of conservatism increases monotonically with his level of conservatism on each political issue. Consequently, a candidate's position on the liberal-conservative dimension is a monotonically decomposible function of his position on each political issue. This in turn implies that a voter's preference for political candidates is folded independent over the multidimensional space determined by the political issues.

A fifth example is found in equity theory. Equity theory is concerned with the study of the "fairness" of a distribution of outcomes or goods. As a model of inequity 
judgments, Anderson and Farkas (1975, see also Anderson, 1976) proposed that perceived inequity is a function of the algebraic expression,

$$
\left[O_{\mathrm{A}} /\left(O_{\mathrm{A}}+O_{\mathrm{B}}\right)\right]-\left[I_{\mathrm{A}} /\left(I_{\mathrm{A}}+I_{\mathrm{B}}\right)\right],
$$

where $O_{\mathrm{A}}, O_{\mathrm{B}}, I_{\mathrm{A}}, I_{\mathrm{B}}$ are the outcomes and inputs for persons A and B, respectively. According to Anderon and Farkas, perceived inequity increases as the value of this expression deviates from zero, although the increase may not be symmetric over positive and negative values. Consequently, perceived equity is single-peaked over the dimension defined by the values of this algebraic expression. This in turn implies that equity judgments are folded additive over the space defined by the dimensions $\left[O_{\mathrm{A}} /\left(O_{\mathrm{A}}+O_{\mathrm{B}}\right)\right]$ and $-\left[I_{\mathrm{A}} /\left(I_{\mathrm{A}}+I_{\mathrm{B}}\right)\right]$. This algebraic expression, incidentally, corresponds to a psychological assumption that equity judgements are determined by the perceived value on a mediating dimension that corresponds to person A's, “... relative deserts minus his relative deservingness" (Anderson \& Farkas, 1975, p. 588).

In a similar manner, Anderson and Farkas' other two candidates for the algebraic basis of inequity judgements,

$$
\begin{aligned}
& {\left[O_{\mathrm{A}} / I_{\mathrm{A}}\right]-\left[O_{\mathrm{B}} / I_{\mathrm{B}}\right]} \\
& {\left[O_{\mathrm{A}} / O_{\mathrm{B}}\right]-\left[I_{\mathrm{A}} / I_{\mathrm{B}}\right]}
\end{aligned}
$$

also predict the existence of a folded additive structure, but over different dimensions.

In general, it may be anticipated that whenever we are dealing with behavior which exhibits single-peakedness, it should be possible to construct situations in which folded additivity, or at least folded independence, should hold. In addition, as Coombs and Avrunin (1977) have pointed out, single-peakedness is a frequently occuring and very general property of behavior. This would suggest that the number of domains in which folded additivity might be found is considerable.

Because of the number of potential applications of folded additive structures, an analysis of the basis of folded additivity should be useful. In the next section, a set of necessary and sufficient tests for there to exist a folded additive or folded independent representation of a finite data set, which varies on two dimensions, is given.

\section{BASIC THEORY}

In this section, $\left\langle A X P, \geqslant_{i}\right\rangle$ will represent a weak ordering, $\geqslant_{i}$, on a two-factor data matrix, with dimensions $A$ and $P$. In general, $\geqslant_{0}$ will represent an empirically derived ordering. Theorem 1 can also be applied to any partial order on $A X P$.

The following definition is made.

DefinItion 1 [Single-peakedness]. Let $D=\left\{d_{1}, d_{2}, \ldots, d_{n}\right\}$ be a set of elements for 
which $\left\langle D, \geqslant_{1}\right\rangle$ has $d_{n} \geqslant_{1} d_{n-1} \geqslant_{1} \cdots \geqslant_{1} d_{1}$, then $\left\langle D, \geqslant_{0}\right\rangle$ is single-peaked over $\langle D, \geqslant 1\rangle$ if

$$
d_{i-k} \text { and } d_{i+m}>_{0} d_{i}
$$

never occurs, where $k, m>0$ and

$$
d_{i}={ }_{0} d_{j} \quad \text { only if } \quad d_{i}={ }_{1} d_{j}{ }^{3}
$$

The most important property of single-peaked functions is that, when compared to $\geqslant_{1}$, the ordering, $\geqslant_{0}$, increases monotonically to a certain point and then decreases monotonically.

LEMmA $1 .\left\langle D, \geqslant_{0}\right\rangle$ is single-peaked over, $\left\langle D, \geqslant_{1}\right\rangle$ if and only if there exist real valued functions $G$ and $F$ on $D$, which preserve the $\geqslant_{0}$ and $\geqslant_{1}$ orderings, respectively, such that the values of the function $G$ are single-peaked over the values of the function $F$.

Proof. Sincef the $G$ and $F$ functions preserve the orderings $\geqslant_{0}$ and $\geqslant_{1}$, the proof is immediate.

DefinITION 2 [Monotone decomposability]. $\left\langle A X P, \geqslant_{1}\right\rangle$ is monotonically decomposable if there exist real-valued functions $F$ on $A X P, r_{1}$ on $A$, and $r_{2}$ on $P$ such that, for all $a, a^{\prime}$ in $A$ and $p, p^{\prime}$ in $P$,

(i) $F(a, p)>F\left(a^{\prime}, p^{\prime}\right)$ iff $(a, p)>_{1}\left(a^{\prime}, p^{\prime}\right)$,

(ii) $F(a, p)=F\left(a^{\prime}, p^{\prime}\right)$ iff $(a, p)={ }_{1}\left(a^{\prime}, p^{\prime}\right)$,

(iii) $F(a, p)=f\left(r_{1}(a), r_{2}(p)\right)$,

where $f$ increases monotonically with each of its arguments.

Definition 3 [Independence]. $\left\langle A X P, \geqslant_{1}\right\rangle$ satisfies independence if we can assign subscripts to the elements of $A$ and $P$ in such a way that,

$$
\left(a_{i-k}, p_{j-m}\right)>_{1}\left(a_{i}, p_{j}\right)
$$

never occurs, where $k+m>0$.

LEMMA 2. $\left\langle A X P, \geqslant_{1}\right\rangle$ is monotonically decomposible if and only if it satisfies independence.

Proof. See Krantz, Luce, Suppes, and Tversky (1971, p. 318).

Given these definitions and lemmas, we can now define folded independence.

${ }^{3}$ In all subscripts assume that $i, j, k, m, q, r \geqslant 0$. 
DEFINITION 4 [Folded independence]. The data matrix $\left\langle A X P, \geqslant_{0}\right\rangle$ is a folded independent structure if there exists an ordering, $\geqslant_{1}$, on $A X P$ such that,

(i) $\left\langle A X P, \geqslant_{0}\right\rangle$ is single-peaked over $\left\langle A X P, \geqslant_{1}\right\rangle$,

(ii) $\left\langle A X P, \geqslant_{1}\right\rangle$ satisfies independence.

If (i) and (ii) above hold, then we say that $\left\langle A X P, \geqslant_{0}\right\rangle$ is folded independent over $\left\langle A X P, \geqslant_{1}\right\rangle$.

An example of a folded independent matrix can be seen in Fig. 2a. Note that this ordering is singlc-peaked over the ordering of the cells in Fig. $2 \mathrm{c}$ and that this second ordering satisfies independence.

The only condition required for folded independence is the subset condition.

DEFINITION 5 [The subset condition]. $\left\langle A X P, \geqslant_{0}\right\rangle$ satisfies the subset condition if we can assign subscripts to the elements of $A$ and $P$ such that

$$
\left(a_{i-q}, p_{j-r}\right) \quad \text { and } \quad\left(a_{i+k}, p_{j+m}\right)>_{0}\left(a_{i}, p_{j}\right)
$$

never occurs, where $q+r>0$ and $k+m>0$.

Note that the matrix in Fig. 2a satisfies the subset condition.

THEOREM 1 [Folded independence theorem]. $\left\langle A X P, \geqslant_{0}\right\rangle$ is a folded independent structure, if and only if it satisfies the subset condition.

Proof of necessity. By folded independence, $\left\langle A X P, \geqslant_{0}\right\rangle$ is single-peaked over some independent system, say, $\left\langle A X P, \geqslant_{1}\right\rangle$. By the definition of independence, we can assign subscripts to the elements of $A$ and $P$ so that,

$$
\left(a_{i+k}, p_{j+m}\right) \geqslant_{1}\left(a_{i}, p_{j}\right) \geqslant_{1}\left(a_{i-q}, p_{j-r}\right),
$$

is never violated. Since the single-peakedness property applies to the entire set $A X P$, it must be also apply to any three-element subset (Arrow, 1963, p. 77). An examination of all possible orderings that are single-peaked over (2.3) will reveal that (2.2) never occurs.

\section{Proof of Sufficiency}

This proof is divided into two parts. Part 1 describes the additive unfolding algorithm which can be used to construct an independent system, $\langle A X P, \geqslant 1\rangle$, from $\left\langle A X P, \geqslant_{0}\right\rangle$. Part 2 shows that this "unfolded" order always satisfies the singlepeakedness and independence requirements.

Part 1. The additive unfolding algorithm. (An example of this algorithm is provided below.)

Step 1: Assign subscripts to elements of $A$ and $P$ such that the subset condition is satisfied. 
Step 2: Determine which elements of $A X P$ are above and below the ideal trace. This is done as follows.

(a) If for some $k+m>0$

$$
\left(a_{i+k}, p_{j+m}\right)>_{0}\left(a_{i}, p_{j}\right)
$$

then let $\left(a_{i}, p_{j}\right)$ be below the ideal trace.

(b) If for some $q+r>0$

$$
\left(a_{i-q}, p_{j-r}\right)>_{0}\left(a_{i}, p_{j}\right)
$$

then let $\left(a_{i}, p_{j}\right)$ be above the ideal trace.

(c) For all elements not assigned by $2 a$ or $2 b$, arbitrarily decide if each of them is above or below the ideal trace.

Step 3: Assign an ordering $\geqslant_{1}$ to the elements of $A X P$ such that,

(a) $\geqslant_{1}$ preserves the $\geqslant_{0}$ ordering for all elements below the ideal trace.

(b) $\geqslant_{1}$ reverses the $\geqslant_{0}$ ordering for all elements above the ideal trace.

(c) All elements above the ideal trace are ordered above all elements below the ideal trace.

Part 2. We first show that the algorithm can always be carried out on a data structure which satisfies the subset condition.

Since $\left\langle A X P, \geqslant_{0}\right\rangle$ satisfies the subset condition, it is immediate that step 1 can always be carried out. To show that step 2 can always be carried out, we need only show that the necessary conditions of $2 a$ and $2 b$ cannot apply to the same element and therefore lead to a contradiction. To show this we assume that the necessary conditions of $2 \mathrm{a}$ and $2 \mathrm{~b}$ are both true. This gives us that for some $k, m, q, r$

$$
\left(a_{i-q}, p_{j-r}\right) \quad \text { and } \quad\left(a_{i+k}, p_{j+m}\right) \geqslant_{0}\left(a_{i}, p_{j}\right)
$$

which violates the subset condition.

Step 3 simply assigns the $\geqslant_{1}$ ordering so that all elements below the ideal trace are increasing on the $\geqslant_{0}$ ordering and all elements above the ideal trace are decreasing on the $\geqslant_{0}$ ordering. This guarantees that $\geqslant_{0}$ is single-peaked over $\geqslant_{1}$.

In order to complete this proof, therefore, we need only show that the constructed system $\left\langle A X P, \geqslant_{1}\right\rangle$ is independent.

To show this, we need to show that

$$
\left(a_{i}, p_{j}\right)>_{1}\left(a_{i+k}, p_{j+m}\right)
$$

cannot occur. This is done as follows. An inspection of the additive unfolding algorithm will reveal that (2.4) can only occur as a result of steps $3 \mathrm{a}$ or $3 \mathrm{~b}$. 
If (2.4) resulted from step $3 \mathrm{a}$, then we must have had $\left(a_{i}, p_{j}\right)$ and $\left(a_{i+k}, p_{j+m}\right)$ below the ideal trace and

$$
\left(a_{i}, p_{j}\right)>_{0}\left(a_{i+k}, p_{j+m}\right)
$$

However, this contradicts $2 b$.

Similarly, if $(2.4)$ resulted from $3 b$, we must have had $\left(a_{i}, p_{j}\right)$ and $\left(a_{i+k}, p_{j+m}\right)$ above the ideal trace and

$$
\left(a_{i+k}, p_{j+m}\right)>_{0}\left(a_{i}, p_{j}\right)
$$

which contradicts $2 \mathrm{a}$.

Theorem 1 tells us that the subset condition is a necessary and sufficient test for folded independence. Furthermore, the additive unfolding algorithm (part 1 of the sufficiency proof of Theorem 1) is used to construct an independent system that $\left\langle A X P, \geqslant_{0}\right\rangle$ is single-peaked over. This algorithm plays a central role in the folded additivity analysis that follows. Therefore, an example of how this algorithm can be used to construct an independent system is given.

Consider the matrix in Fig. 2a. Step 1 of the additive unfolding algorithm requires that the rows and columns of this matrix be permuted so that (2.2) of the subset condition is satisfied. In the case of Fig. $2 a$, it can be verified that this was already done. If step 1 cannot be carried out then Fig. 2 a could not be a folded independent structure.

In step 2 of the algorithm, we examine each row and column to determine which cells must be above or below the ideal trace. For a fully ordered data matrix this can be done as follows. For each row/column, find the cell which is highest on the behavioral ordering in that row/column. Cells which are positionally to the left of/below this cell will be below the ideal trace. On the other hand, those cells which are positionally to the right of/above the highest ordered cell in each row/column will be above the ideal trace. Since $\left(a_{1}, p_{3}\right)$ and $\left(a_{2}, p_{1}\right)$ are the highest ordered in their row and column, step $2 \mathrm{c}$ is applied to arbitrarily determine whether each is above or below. For now assume that $\left(a_{1}, p_{3}\right)$ is above and $\left(a_{2}, p_{1}\right)$ is below the ideal trace.

In Fig. $2 b$, those cells which step 2 marked as below the ideal trace on the unfolded order are indicated by $a \downarrow$ and those cells which are above the ideal trace are indicated by a $\uparrow$.

Step 3 of the algorithm requires that the unfolded order be strictly monotone with the behavioral order for all cells below the ideal trace on the unfolded order, and inversely monotone for all cells above the ideal trace on the unfolded order. This gives us the unfolded order in Fig. 2c.

The reader can verify that if we had made different decisions about $\left(a_{1}, p_{3}\right)$ and $\left(a_{2}, p_{1}\right)$ we would still have constructed an independent system.

In the following analysis, we are going to limit the discussion to empirical orderings with the following properties, 


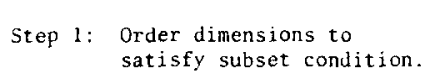

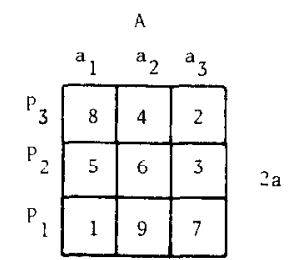
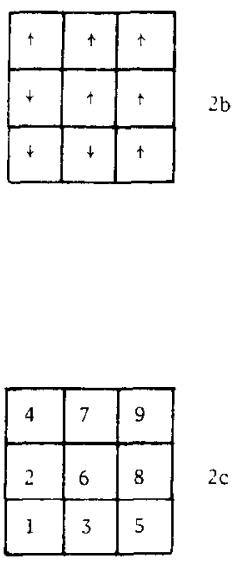

FIG. 2. An example of the Additive Unfolding Algorithm. The cell values in matrix $2 \mathrm{c}$ were derived from the empirical ordering in matrix $2 a$.

(i) for all $a, a^{\prime}$ in $A$, there exists a $p$ in $P$, such that

$$
\left(a^{\prime}, p\right) \neq_{0}(a, p),
$$

(ii) for all $p, p^{\prime}$ in $P$, there exists an $a$ in $A$, such that

$$
\left(a, p^{\prime}\right) \neq_{0}(a, p) .
$$

This restriction states, in effect, that we are limiting our discussion to data structures in which the behavioral ordering, $\geqslant_{0}$, is not completely indifferent to different levels of the independent variables.

LEMMA 3. If $\left\langle A X P, \geqslant_{0}\right\rangle$ is restricted as above and is folded-independent over $\left\langle A X P, \geqslant_{1}\right\rangle$, then for all $a_{i}$ in $A$ and $P_{j}$ in $P$

$$
\left(a_{i+k}, p_{j}\right) \neq{ }_{1}\left(a_{i}, p_{i}\right)
$$


and

$$
\left(a_{i}, p_{j+m}\right) \neq_{1}\left(a_{i}, p_{j}\right)
$$

Proof. By single-peakedness

$$
\left(a_{i+k}, p_{j+m}\right)={ }_{1}\left(a_{i}, p_{j}\right) \text { implies }\left(a_{i+k}, p_{j+m}\right)={ }_{0}\left(a_{i}, p_{j}\right) .
$$

Lemma 3 applies the contrapositive of this to the restriction.

Generally speaking, a folded independent system, $\left\langle A X P, \geqslant_{0}\right\rangle$, is single-peaked over a number of different indepedent systems. Therefore we can define $W\left(A X P, \geqslant_{0}\right)$ as the set of all independent systems that $\left\langle A X P, \geqslant_{0}\right\rangle$ is single-peaked over.

COROLLARY 1. If $\left\langle A X P, \geqslant_{1}\right\rangle$ was constructed from $\left\langle A X P, \geqslant_{0}\right\rangle$ using the additive unfolding algorithm, then

$$
\left\langle A X P, \geqslant_{1}\right\rangle \in W\left(A X P, \geqslant_{0}\right) .
$$

Since step 2 of the algorithm permits us to make some arbitrary decisions about the unfolded order, there will usually exist several independent systems that can be constructed using the algorithm. Let $U\left(A X P, \geqslant_{0}\right)$ be the set of all independent systems that can be constructed from $\left\langle A X P, \geqslant_{0}\right\rangle$ by making different decisions in step $2 \mathrm{c}$. We then have the following corollary.

COROLlaRY 2. If $\left\langle A X P, \geqslant_{1}\right\rangle$ and $\left\langle A X P, \geqslant_{2}\right\rangle$ are elements of $U\left(A X P, \geqslant_{0}\right)$, then $\left\langle A X P, \geqslant_{1}\right\rangle$ and $\left\langle A X P, \geqslant_{2}\right\rangle$ have the same order on $A$ and $P$. That is

$$
(a, p) \geqslant_{1}\left(a^{\prime}, p\right) \quad \text { iff } \quad(a, p) \geqslant_{2}\left(a^{\prime}, p\right)
$$

and

$$
(a, p) \geqslant_{1}\left(a, p^{\prime}\right) \quad \text { iff }(a, p) \geqslant_{2}\left(a, p^{\prime}\right) .
$$

Proof. From Part 2 of the sufficiency proof of Theorem 1, we know that the additive unfolding algorithm will always construct an order such that (2.4) never occurs. Corollary 2 is an immediate consequence of this.

It is a characteristic of single-peaked functions that if $\left\langle A X P, \geqslant_{0}\right\rangle$ is single-peaked over $\left\langle A X P, \geqslant_{1}\right\rangle$, then $\left\langle A X P, \geqslant_{0}\right\rangle$ is also single-peaked over the system with the reverse ordering $\left\langle A X P, \leqslant_{1}\right\rangle$. Furthermore, if $\left\langle A X P, \geqslant_{1}\right\rangle$ is independent, so is $\langle A X P, \leqslant 1\rangle$.

Let $V\left(A X P, \geqslant_{0}\right)$ be the set of all systems $\left\langle A X P, \leqslant_{1}\right\rangle$ for which $\left\langle A X P, \geqslant_{1}\right\rangle$ is in $U\left(A X P, \geqslant_{0}\right)$. We have the following corollary.

COROllary 3. $U\left(A X P, \geqslant_{0}\right) \cup V\left(A X P, \geqslant_{0}\right) \subseteq W\left(A X P, \geqslant_{0}\right)$.

Corollary 3 tells us that all the weakly ordered systems that can be constructed by 
the additive unfolding algorithms, and those with the reverse ordering, make up a subset of $W\left(A X P, \geqslant_{0}\right)$. Lemmas 4 and 5 and Theorem 2 extends this analysis by showing that these two sets are in fact equivalent.

LEMMA 4. If $\left\langle A X P, \geqslant_{0}\right\rangle$ is folded independent over $\left\langle A X P, \geqslant_{1}\right\rangle$ and $\left\langle A X P, \geqslant_{2}\right\rangle$ and we assign subscripts to the elements of $A$ and $P$ to be monotone increasing with $\geqslant_{1}$, then ${ }^{4}$ for $k, m>0$

$$
\left(a_{i+k}, p_{j+m}\right)>_{1}\left(a_{i+k}, p_{j}\right),\left(a_{i}, p_{j+m}\right)>_{1}\left(a_{i}, p_{j}\right)
$$

implies one of the following:

$$
\left(a_{i+k}, p_{j+m}\right)>_{2}\left(a_{i+k}, p_{j}\right),\left(a_{i}, p_{j+m}\right)>_{2}\left(a_{i}, p_{j}\right)
$$

or

$$
\left(a_{i}, p_{j}\right)>_{2}\left(a_{i+k}, p_{j}\right),\left(a_{i}, p_{j+m}\right)>_{2}\left(a_{i+k}, p_{j+m}\right) .
$$

Proof. Assume that the above is not true, then we have

$$
\left(a_{i+k}, p_{j+m}\right)>_{1}\left(a_{i}, p_{j+m}\right),\left(a_{i+k}, p_{j}\right)>_{1}\left(a_{i}, p_{j}\right)
$$

and, from the independence of $\left\langle A X P, \geqslant_{2}\right\rangle$, one of the following,

$$
\left(a_{i+k}, p_{j}\right)>_{2}\left(a_{i+k}, p_{j+m}\right),\left(a_{i}, p_{j}\right)>_{2}\left(a_{i}, p_{j+m}\right)
$$

or

$$
\left(a_{i}, p_{j+m}\right)>_{2}\left(a_{i+k}, p_{j+m}\right),\left(a_{i}, p_{j}\right)>_{2}\left(a_{i+k}, p_{j}\right) .
$$

Since $\left\langle A X P, \geqslant_{0}\right\rangle$ is single-peaked over $\left\langle A X P, \geqslant_{1}\right\rangle,(2.6)$ implies

$$
\begin{aligned}
& \left(a_{i+k}, p_{j}\right),\left(a_{i}, p_{j+m}\right)>_{0}\left(a_{i+k}, p_{j+m}\right) \\
& \left(a_{i+k}, p_{j}\right),\left(a_{i}, p_{j+m}\right)>_{0}\left(a_{i}, p_{j}\right) .
\end{aligned}
$$

Since $\left\langle A X P, \geqslant_{0}\right\rangle$ is single-peaked over $\left\langle A X P, \geqslant_{2}\right\rangle,(2.7)$ and (2.8) each imply that

$$
\begin{aligned}
& \left(a_{i+k}, p_{j+m}\right),\left(a_{i}, p_{j}\right)>_{0}\left(a_{i+k}, p_{j}\right) \\
& \left(a_{i+k}, p_{j+m}\right),\left(a_{i}, p_{j}\right)>_{0}\left(a_{i}, p_{j+m}\right) .
\end{aligned}
$$

(2.10) contradicts (2.9).

This last result, combined with Corollary 3 , tells us that if $\left\langle A X P, \geqslant_{0}\right\rangle$ is singlepeaked over an independent system $\left\langle A X P, \geqslant_{1}\right\rangle$, then the ordering that $\geqslant_{1}$ induces on the elements in $A$ and $P$ must be the same as for the members of either $U\left(A X P, \geqslant_{0}\right)$ or $V\left(A X P, \geqslant_{0}\right)$.

\footnotetext{
${ }^{4}$ Any inequality of the form $a>b, c>d$ should be read as $a>b$ and $c$, and $b$ and $c>d$.
} 
Lemma 5. Assume that the order of the elements in $A$ and $P$ for the system $\left\langle A X P, \geqslant_{1}\right\rangle$ is the same as that for $U\left(A X P, \geqslant_{0}\right)$. Then $\left\langle A X P, \geqslant_{1}\right\rangle \in U\left(A X P, \geqslant_{0}\right)$.

Proof. To show this, it is sufficient to show that each step in the additive unfolding algorithm is implied by the fact that $A$ and $P$ are already ordered.

Step 1 assigns subscripts to the elements of $A$ and $P$ so that the order on $A$ and $P$ is consistent with that of $\left\langle A X P, \geqslant_{1}\right\rangle$. This is equivalent to assuming that the ordering on $A$ and $P$ is given.

Step $2 \mathrm{a}$ is shown to be implied as follows. From our ordering assumption we have

$$
\left(a_{i+k}, p_{j+m}\right)>_{1}\left(a_{i}, p_{j}\right)
$$

Since, from $2 \mathrm{a}$

$$
\left(a_{i+k}, p_{j+m}\right)>_{0}\left(a_{i}, p_{j}\right)
$$

we know that $\geqslant_{0}$ is monotonically increasing from $\left(a_{i}, p_{j}\right)$ to $\left(a_{i+k}, p_{j+m}\right)$ on $\geqslant_{1}$. Consequently, $\left(a_{i}, p_{j}\right)$ must be below the peak, i.e., below the ideal trace. The proof of $2 \mathrm{~b}$ is similar to that of $2 \mathrm{a}$ and need not be repeated here.

Finally, step 3 is based on a basic property of single-peaked functions, namely, that relative to the unfolded order, the behavioral ordering should increase monotonically below the peak and decrease monotonically above the peak.

THEOREM 2 [Uniqueness of the unfolded independent order].

$$
W\left(A X P, \geqslant_{0}\right)=U\left(A X P, \geqslant_{0}\right) \cup V\left(A X P, \geqslant_{0}\right) .
$$

Proof. Lemma 4 tells us that in order for $\left\langle A X P, \geqslant_{0}\right\rangle$ to be single-peaked over $\left\langle A X P, \geqslant_{1}\right\rangle$, the order that $\geqslant_{1}$ induces on the elements of $A$ and $P$ must be compatible with the order on $A$ and $P$ for the members of either $U\left(A X P, \geqslant_{0}\right)$ or $V\left(A X P, \geqslant_{0}\right)$. Lemma 5 states that if $\left\langle A X P, \geqslant_{1}\right\rangle$ orders the elements of $A$ and $P$ in such a way that their order is compatible with the structures in $U\left(A X P, \geqslant_{0}\right)$, then $\left\langle A X P, \geqslant_{1}\right\rangle$ is an element of $U\left(A X P, \geqslant_{0}\right)$. Finally, by the definition of $V\left(A X P, \geqslant_{0}\right),\left\langle A X P, \geqslant_{1}\right\rangle$ is an element of $U\left(A X P, \geqslant_{0}\right)$ if and only if $\left\langle A X P, \leqslant_{1}\right\rangle$ is an element of $V\left(A X P, \geqslant_{0}\right)$. Combining these three results, we find that a folded independent system, $\left\langle A X P, \geqslant_{0}\right\rangle$, is single-peaked over an independent system, $\left\langle A X P, \geqslant_{1}\right\rangle$, if and only if

$$
\left\langle A X P, \geqslant_{1}\right\rangle \in U\left(A X P, \geqslant_{0}\right) \quad \text { or } \quad\left\langle A X P, \geqslant_{1}\right\rangle \in V\left(A X P, \geqslant_{0}\right) .
$$

Together with Corollary 3 , this gives us

$$
W\left(A X P, \geqslant_{0}\right)=U\left(A X P, \geqslant_{0}\right) \cup V\left(A X P, \geqslant_{0}\right) .
$$

We now turn to the case of folded additivity.

DEFINITION 6 [Additivity]. $\left\langle A X P, \geqslant_{1}\right\rangle$ has an additive representation if there 
exists a function $F$ on $A X P, r_{1}$ on $A$, and $r_{2}$ on $P$ such that for all $a, a^{\prime}$ in $A$ and $p, p^{\prime}$ in $P$,

(i) $(a, p)>_{1}\left(a^{\prime}, p^{\prime}\right)$ implies $F(a, p)>F\left(a^{\prime}, p^{\prime}\right)$,

(ii) $(a, p)={ }_{1}\left(a^{\prime}, p^{\prime}\right)$ implies $F(a, p)=F\left(a^{\prime}, p^{\prime}\right)$,

(iii) $F(a, p)=r_{1}(a)+r_{2}(p)$.

LEMMA 6. If $\left\langle A X P, \geqslant_{1}\right\rangle$ is additive, then it is also satisfies independence.

Proof. This lemma follows directly from the fact that an additive structure is a type of monotonically decomposible structure.

Definition 7 [Folded additivity]. The data matrix $\left\langle A X P, \geqslant_{0}\right\rangle$ is a folded additive structure if there exists a weak ordering, $\geqslant_{1}$, on $A X P$ such that

(i) $\left\langle A X P, \geqslant_{0}\right\rangle$ is single-peaked over $\left\langle A X P, \geqslant_{1}\right\rangle$,

(ii) $\left\langle A X P, \geqslant_{1}\right\rangle$ is an additive structure.

THEOREM 3 [Folded additivity theorem]. $\left\langle A X P, \geqslant_{0}\right\rangle$ is single-peaked over an additive system $\left\langle A X P, \geqslant_{1}\right\rangle$ if and only if $\left\langle A X P, \geqslant_{1}\right\rangle \in W\left(A X P, \geqslant_{0}\right)$.

Proof. Recall that if $\left\langle A X P, \geqslant_{1}\right\rangle$ is additive, it is also independent. So if $\left\langle A X P, \geqslant_{1}\right\rangle$ is additive, and $\left\langle A X P, \geqslant_{0}\right\rangle$ is single-peaked over $\left\langle A X P, \geqslant_{1}\right\rangle$, then $\left\langle A X P, \geqslant_{1}\right\rangle \in W\left(A X P, \geqslant_{0}\right)$. On the other hand, if the additive system $\left\langle A X P, \geqslant_{1}\right\rangle$ is in $W\left(A X P, \geqslant_{0}\right)$, then $\left\langle A X P, \geqslant_{0}\right\rangle$ is by definition single-peaked over $\left\langle A X P, \geqslant_{1}\right\rangle$.

There already exist several techniques for determing whether or not a structure is additive (Lehner \& Noma, 1980; Scott, 1964). Therefore, to test for folded additivity, we need only apply one of these techniques to all the elements of $W\left(A X P, \geqslant_{0}\right)$. In addition, any of these methods can be used to assign numeric scale values to the $A$ and $P$ elements. Consequently, a folded additive representation can be constructed. In addition it can be shown that if $A$ and $P$ have $m$ and $n$ elements, respectively, and $\left\langle A X P, \geqslant_{0}\right\rangle$ is fully ordered, then the maximum number of different independent orders in $W\left(A X P, \geqslant_{0}\right)$ is $2^{\min (m, n)}$. Therefore, an exhaustive search of $W\left(A X P, \geqslant_{0}\right)$ is usually feasible.

There is an interesting property associated with the uniqueness of the unfolded additive solution. For an additive ordering on $\left\langle A X P, \geqslant_{1}\right\rangle$ it has been shown [see McClelland \& Coombs, 1975] that the solution space of scale values for the $A$ and $P$ elements form a convex set. In the case of a folded additive system, however, there will usually exist several additive orders in $W\left(A X P, \geqslant_{0}\right)$. Consequently, the solution space of scale values for the $A$ and $P$ elements will form a set of convex sets. 


\section{Discussion}

Briefly summarized, the results of the last section indicate that a behavioral ordering satisfies folded independence if and only if the subset condition (definition 5) is satisfied. Furthermore, the additive unfolding algorithm can be used to find all independent systems that unfold the behavioral ordering. Consequently, folded additivity is satisfied if and only if one of the independent systems constructed by the additive unfolding algorithm has an additive representation.

This approach differs radically from the statistical use of interaction terms to explain variance in a factorial design. More often than not, these statistical interactions are purely mathematical constructs that are not easily interpreted. Folded additivity, on the other hand, provides a mathematical model which leads directly to a certain class of psychological interpertations, viz., single-peakedness over an additive composition.

Finally, it should be noted that the present theory does not assume that the data are in numerical form, only a set of qualitative tests are presented. In addition, given that these tests are satisfied, it is shown how a numerical representation can be constructed. Consequently, the present theory is an example of a fundamental measurement model.

\section{REFERENCES}

Anderson, N. H. Equity judgments as information integration. Journal of Personality and Social Psychology, 1976, 33(3), 291-299.

Anderson, N. H., \& Farkas, A. J. Integration theory applied to models of inequity. Personality and Social Psychology Bulletin, 1975, 1, 588-591.

Aronson, E., Willerman, B., \& Floyd, J. The effect of a pratfall on increasing interpersonal attractiveness. Psychonomic Science, 1966, 4, 227-228.

Arrow, K. J. Social choice and individual values. New Haven, Conn.: Cowles Foundation, 1963.

BARGH, J. A., \& COHEN, J. L. Mediating factors in the arousal-performance relationship. Motivation and Emotion, 1978, 2, 243-257.

Coombs, C. H. A method for the study of interstimulus similaity. Psychometrika, 1954, 19, 183-194.

Coombs, C. H. A theory of data. Nzw York: Wiley, 1964.

Coombs, C. H. Portfolio theory: a theory of risky decision making. La decision, Paris, France, Centre Nationale de la Rescherche Scientifique, 1969.

Coombs, C. H. Portfolio theory and the measurement of risk. In Schwartz, S. \& Kaplan, M. (eds.), Human judgement and decision processes. Academic Press, 1975.

Coombs, C. H. \& Avrunin, G. S. Single-peaked functions and the theory of preference. Psychological Review, 1977, 84, 216-230.

Coombs, C. H., \& Huang, C. H. Polynomial psychophysics of risk. Journal of Mathematical Psychology, 1970, 7, 317-338.

COOMBs, C. H., \& LEHNER, P. E. A comparison of some alternative sets of variables for the theory of risk: I. A polynomial psychophysical model. Journal of Experimental Psychology: Human Perception and Performance, 1980.

CoOmbs, C. H., \& Meyer, D. E. Risk preference in coin-toss games. Journal of Mathematical Psychology, 1969, 6, 514-527.

INNES, J. M., \& Young, R. F. The effect of presence of an audience, evaluation apprehension, and objective self-awareness on learning. Journal of Experimental Social Psychology, 1975, 11, 35-42. 
Katahn, M., Blanton, R. L., \& Gipson, M. T. Speed and amplitude of response as a function of anxiety and degree of conflict. Journal of Experimental Research in Personality, 1967, 2, 169-172.

Krantz, D. H., Luce, R. D., Suppes, P., \& TVersky, A. Foundations of measurement (Vol. 1). New York: Academic Press, 1971.

LEHNER, P. E. A comparison of Portfolio theory and weighted utility models of risky decision making. Organizational Behavior and Human Performance, 1980, 26, 238-249.

LEHNER, P. E., \& NOMA, E. A new solution to the problem of finding all numerical solutions to ordered metric structures. Psychometrika, 1980, 45(1), 135-137.

Liebling, B. A., \& SHAVER, P. Evaluation, self-awareness, and task performance. Journal of Experimental Psychology, 1973, 9, 297-306.

MARTENS, R., \& LANDERS, D. M. Evaluation potential as a determinant of coaction effects. Journal of Experimental Social Psychology, 1972, 8, 347-359.

MCClelLAND, G. H., \& COOMBS, C. H. ORDMET, a general algorithm for constructing all numerical solutions to ordered metric structures. Psychometrika, 1975, 40, 269-290.

Scotr, D. Measurement models and linear inequalities. Journal of Mathematical Psychology, 1964, 1. 233-248.

SORCE, J.. \& Fouts, G. Level of motivation in social facilitation of a simple task. Perceptual and Motor Skills, 1973, 37, 567-572.

STENNET, R. G. The relationship between performance level to level of arousal. Journal of Experimental Psychology, 1957, 54, 54-61.

\section{REFERENCE NOTES}

1. Berger, T. A. A polynomial theory of risk (MMPP 74-4). Michigan Mathematical Psychology Program technical report, 1974.

RECEIVED: February 7, 1980 